\title{
FRACTIONAL DERIVATIVE OPERATOR FOR P-VALENT FUNCTIONS WITH NEGATIVE COEFFICIENTS
}

\author{
M. Aljarrah, A. Aljarah, M. Darus
}

Abstract. In this paper, a differential operator $D_{p, \mu, \lambda, \sigma}^{m}(\alpha, \beta) f(z)$ defined in the open unit disc $U=\{z \in \mathbb{C}:|z|<1\}$ is introduced. By using this operator, we introduce a new subclass of analytic functions $G_{n}^{p}(\alpha, \beta, \mu, \lambda, \gamma, \delta)$. Moreover, we discuss coefficient inequality, Hadamand product, growth and distortion theorems, closure theorems, radii of close-to-convexity, starlikeness, convexity and integral operators. Furthermore, we give an application involving fractional calculus for functions in $G_{n}^{p}(\alpha, \beta, \mu, \lambda, \gamma, \delta)$.

\section{Mathematics Subject Classification: 30C45.}

Keywords: Analytic functions, p-valent functions, differential operator, fractional calculus.

\section{INTRODUCTION AND PRELIMINARIES}

Let $\mathcal{A}_{p}$ be the class of function $f(z)$ of the form

$$
f(z)=z^{p}-\sum_{k=p+1}^{\infty} a_{k} z^{k} \quad\left(a_{k} \geq 0, z \in \mathbb{U}, p \in \mathbb{N}=1,2,3, \ldots\right)
$$

which are analytic in the unit disc $\mathbb{U}=\{z \in \mathbb{C}:|z|<1\}$. For a function $f$ in $\mathcal{A}_{p}$, we define the following differential operator

$$
\begin{gathered}
D_{p, \mu, \lambda, \sigma}^{0}(\alpha, \beta) f(z)=f(z) \\
D_{p, \mu, \lambda, \sigma}^{1}(\alpha, \beta) f(z)=\left(\frac{\mu+\lambda-(\beta-\sigma)(\lambda-\alpha)}{\mu+\lambda}\right) f(z)+\left(\frac{(\beta-\sigma)(\lambda-\alpha)}{\mu+\lambda}\right) z f^{\prime}(z),
\end{gathered}
$$

and for $m=1,2,3, \cdots$

$$
D_{p, \mu, \lambda, \sigma}^{m}(\alpha, \beta) f(z)=D_{p, \mu, \lambda, \sigma}\left(D_{p, \mu, \lambda, \sigma}^{M-1}(\alpha, \beta) f(z)\right) .
$$


If $f$ is given by (1), then from (4) we get

$$
D_{p, \mu, \lambda, \sigma}^{m}(\alpha, \beta) f(z)=z^{p}+\sum_{k=p+1}^{\infty}\left(\frac{\mu+\lambda+(k-p)(\beta-\sigma)(\lambda-\alpha)}{\mu+\lambda}\right)^{m} a_{k} z^{k},
$$

for $f \in \mathcal{A}_{p}, \sigma, \alpha \geq 0, \beta, \mu, \lambda>0, \lambda \neq \alpha, p \in \mathbb{N}$ and $k \in \mathbb{N}_{0}=\mathbb{N} \cup\{0\}$.

We observe that the generalized differential operator $D_{p, \mu, \lambda, \sigma}^{m}(\alpha, \beta) f(z)$ reduces to several other differential operators considered earlier for different choices of $\mu, \lambda, \sigma, \alpha$ and $\beta$ :

(i) $D_{p, \mu, \lambda, \sigma}^{m}(\alpha, \beta) f(z)$ when $p=1$, we have the operator introduced and studied by Amourah and Yousef [1].

(ii) $D_{1,0,1, \sigma}^{m}(\alpha, \beta) f(z)$, we have the operator introduced and studied by Aljarah and Darus [2].

(iii) $D_{1-\lambda, \lambda, \sigma}^{m}(\alpha, \beta) f(z)=z+\sum_{n=2}^{\infty}[1+(n-1)(\lambda-\alpha)(\beta-\sigma)]^{m} a_{n} z^{n}$ was introduced and studied by Ramadan and Darus [4];

(iv) $D_{1-\lambda, \lambda, 0}^{m}(\alpha, \beta) f(z)=z+\sum_{n=2}^{\infty}[1+(n-1)(\lambda-\alpha) \beta]^{m} a_{n} z^{n}$ was introduced and studied by Darus and Ibrahim [3];

(v) $D_{\mu, \lambda, 0}^{m}(0,1) f(z)=z+\sum_{n=2}^{\infty}\left[\frac{\mu+\lambda n}{\mu+\lambda}\right]^{m} a_{n} z^{n}$ was introduced and studied by Swamy [7];

(vi) $D_{1-\lambda, \lambda, 0}^{m}(0,1) f(z)=z+\sum_{n=2}^{\infty}[1+(n-1) \lambda]^{m} a_{n} z^{n}$ was introduced by AlOboudi [8]; [6].

$$
\text { (vii) } D_{0,1,0}^{m}(0,1) f(z)=z+\sum_{n=2}^{\infty} n^{m} a_{n} z^{n} \text { was introduced and studied by Salagean }
$$

Another differential that might be of interests can be seen in [5].

Let $G_{n}^{p}(\alpha, \beta, \mu, \lambda, \gamma, \delta)$ denote the subclass of $\mathcal{A}_{p}$ consisting of functions $f$ which satisfy

$\operatorname{Re}\left\{z \frac{\gamma z^{2}\left(D_{p, \mu, \lambda, \sigma}^{m}(\alpha, \beta) f(z)\right)^{\prime \prime \prime}+(2 \gamma+1) z\left(D_{p, \mu, \lambda, \sigma}^{m}(\alpha, \beta) f(z)\right)^{\prime \prime}+\left(D_{p, \mu, \lambda, \sigma}^{m}(\alpha, \beta) f(z)\right)^{\prime}}{\gamma z^{2}\left(D_{p, \mu, \lambda, \sigma}^{m}(\alpha, \beta) f(z)\right)^{\prime \prime}+z\left(D_{p, \mu, \lambda, \sigma}^{m}(\alpha, \beta) f(z)\right)^{\prime}}\right\}>\delta$

where $D_{p, \mu, \lambda, \sigma}^{m}(\alpha, \beta) f(z)$ is given by $(5), \gamma(0 \leq \gamma \leq 1), \delta(0 \leq \delta<1)$ and for all $z \in U$.

We note that 
M. Aljarrah, A. Aljarah, M. Darus - Fractional derivative operator ...

(i) $G_{n}^{p}(\alpha, \beta, 0,0, \gamma, \delta)$ was introduced by Xiao-Fei and An-Ping [9];

(ii) $G_{n}^{1}(\alpha, \beta, 0,0, \gamma, \delta)$ was introduced by Kamali and Akbulut [10].

\section{Coefficient Inequality for the Class $G_{n}^{p}(\alpha, \beta, \mu, \lambda, \gamma, \delta)$}

We begin with our first result as follows:

Theorem 1. A function $f \in \mathcal{A}$ is in the class $G_{n}^{p}(\alpha, \beta, \mu, \lambda, \gamma, \delta)$ if and only if

$$
\sum_{k=p+1}^{\infty} k(k-\delta)[\gamma k-\gamma+1]\left[\frac{\mu+\lambda+(k-p)(\beta-\sigma)(\lambda-\alpha)}{\mu+\lambda}\right]^{m} a_{k} \leq p(p-\delta)[\gamma p-\gamma+1] \text {. }
$$

The result (7) is sharp.

Proof. Assume that $f \in G_{n}^{p}(\alpha, \beta, \mu, \lambda, \gamma, \delta)$. Then we find from (6) that

$$
\operatorname{Re}\left\{\frac{p^{2}[\gamma p-\gamma+1]-\sum_{k=p+1}^{\infty} k^{2}[\gamma k-\gamma+1]\left[\frac{\mu+\lambda+(k-p)(\beta-\sigma)(\lambda-\alpha)}{\mu+\lambda}\right]^{m} a_{k} z^{k-p}}{p[\gamma p-\gamma+1]-\sum_{k=p+1}^{\infty} k[\gamma k-\gamma+1]\left[\frac{\mu+\lambda+(k-p)(\beta-\sigma)(\lambda-\alpha)}{\mu+\lambda}\right]^{m} a_{k} z^{k-p}}\right\}>\delta .
$$

If we choose $z$ to be the real and let $z \rightarrow 1^{-}$, we get

$$
\begin{aligned}
& p^{2}[\gamma p-\gamma+1]-\sum_{k=p+1}^{\infty} k^{2}[\gamma k-\gamma+1]\left[\frac{\mu+\lambda+(k-p)(\beta-\sigma)(\lambda-\alpha)}{\mu+\lambda}\right]^{m} a_{k} \\
& \geq \delta p[\gamma p-\gamma+1]-\sum_{k=p+1}^{\infty} \delta k[\gamma k-\gamma+1]\left[\frac{\mu+\lambda+(k-p)(\beta-\sigma)(\lambda-\alpha)}{\mu+\lambda}\right]^{m} a_{k}
\end{aligned}
$$

or

$$
\sum_{k=p+1}^{\infty} k(k-\delta)[\gamma k-\gamma+1]\left[\frac{\mu+\lambda+(k-p)(\beta-\sigma)(\lambda-\alpha)}{\mu+\lambda}\right]^{m} a_{k} \leq p(p-\delta)[\gamma p-\gamma+1],
$$

which is equivalent to (7). Conversely, assume that (7) is true. Then we have

$$
\begin{aligned}
&\left|z \frac{\gamma z^{2}\left(D_{p, \mu, \lambda, \sigma}^{m}(\alpha, \beta) f(z)\right)^{\prime \prime \prime}+(2 \gamma+1) z\left(D_{p, \mu, \lambda, \sigma}^{m}(\alpha, \beta) f(z)\right)^{\prime \prime}+\left(D_{p, \mu, \lambda, \sigma}^{m}(\alpha, \beta) f(z)\right)^{\prime}}{\gamma z^{2}\left(D_{p, \mu, \lambda, \sigma}^{m}(\alpha, \beta) f(z)\right)^{\prime \prime}+z\left(D_{p, \mu, \lambda, \sigma}^{m}(\alpha, \beta) f(z)\right)^{\prime}}-1\right|= \\
&\left|\frac{p(p-1)[\gamma p-\gamma+1]-\sum_{k=p+1}^{\infty} k(k-1)[\gamma k-\gamma+1]\left[\frac{\mu+\lambda+(k-p)(\beta-\sigma)(\lambda-\alpha)}{\mu+\lambda}\right]^{m} a_{k} z^{k-p}}{p[\gamma p-\gamma+1]-\sum_{k=p+1}^{\infty} k[\gamma k-\gamma+1]\left[\frac{\mu+\lambda+(k-p)(\beta-\sigma)(\lambda-\alpha)}{\mu+\lambda}\right]^{m} a_{k} z^{k-p}}\right| \leq
\end{aligned}
$$


M. Aljarrah, A. Aljarah, M. Darus - Fractional derivative operator ...

$$
\frac{\sum_{k=p+1}^{\infty} k(k-1)[\gamma k-\gamma+1]\left[\frac{\mu+\lambda+(k-p)(\beta-\sigma)(\lambda-\alpha)}{\mu+\lambda}\right]^{m} a_{k}-p(p-1)[\gamma p-\gamma+1]}{p[\gamma p-\gamma+1]-\sum_{k=p+1}^{\infty} k[\gamma k-\gamma+1]\left[\frac{\mu+\lambda+(k-p)(\beta-\sigma)(\lambda-\alpha)}{\mu+\lambda}\right]^{m} a_{k}} \leq 1-\delta
$$

This implies that $f \in G_{n}^{p}(\alpha, \beta, \mu, \lambda, \gamma, \delta)$. The result (7) is sharp for the function

$$
f(z)=z^{p}-\frac{p(p-\delta)[\gamma p-\gamma+1]}{k(k-\delta)[\gamma k-\gamma+1]\left[\frac{\mu+\lambda+(k-p)(\beta-\sigma)(\lambda-\alpha)}{\mu+\lambda}\right]^{m}} z^{k}(k \geq p+1) .
$$

\section{HadAmand Product}

Definition 1. Let $(f * g)(z)$ denote the Hadamand product (Convolution) of two functions

$$
f(z)=z^{p}-\sum_{k=p+1}^{\infty} a_{k} z^{k}\left(a_{k} \geq 0, n, p \in \mathbb{N} .\right)
$$

and

$$
g(z)=z^{p}-\sum_{k=p+1}^{\infty} b_{k} z^{k}\left(b_{k} \geq 0, n, p \in \mathbb{N}\right)
$$

that is

$$
(f * g)(z)=z^{p}-\sum_{k=p+1}^{\infty} a_{k} b_{k} z^{k} .
$$

Theorem 2. If $f(z), g(z) \in G_{n}^{p}(\alpha, \beta, \mu, \lambda, \gamma, \delta)$, then $(f * g)(z) \in G_{n}^{p}(\alpha, \beta, \mu, \lambda, \gamma, \chi)$ where

$\chi=p\left[1-\frac{(p-\delta)^{2}[\gamma p-\gamma+1]}{(p+1)(p+1-\delta)^{2}[\gamma k-\gamma+1]\left[\frac{\mu+\lambda+(\beta-\sigma)(\lambda-\alpha)}{\mu+\lambda}\right]^{m}-p(p-\delta)^{2}[\gamma p-\gamma+1]}\right]$.

Proof. From Theorem 1, we have

$$
\sum_{k=p+1}^{\infty} \frac{k(k-\delta)[\gamma k-\gamma+1]\left[\frac{\mu+\lambda+(k-p)(\beta-\sigma)(\lambda-\alpha)}{\mu+\lambda}\right]^{m}}{p(p-\delta)[\gamma p-\gamma+1]} a_{k} \leq 1
$$


M. Aljarrah, A. Aljarah, M. Darus - Fractional derivative operator ...

and

$$
\sum_{k=p+1}^{\infty} \frac{k(k-\delta)[\gamma k-\gamma+1]\left[\frac{\mu+\lambda+(k-p)(\beta-\sigma)(\lambda-\alpha)}{\mu+\lambda}\right]^{m}}{p(p-\delta)[\gamma p-\gamma+1]} b_{k} \leq 1
$$

We have to find the largest $\chi$ such that

$$
\sum_{k=p+1}^{\infty} \frac{k(k-\chi)[\gamma k-\gamma+1]\left[\frac{\mu+\lambda+(k-p)(\beta-\sigma)(\lambda-\alpha)}{\mu+\lambda}\right]^{m}}{p(p-\chi)[\gamma p-\gamma+1]} a_{k} b_{k} \leq 1 .
$$

From (10) and by means of Cauch-Schwarz inequality, we have

$$
\sum_{k=p+1}^{\infty} \frac{k(k-\delta)[\gamma k-\gamma+1]\left[\frac{\mu+\lambda+(k-p)(\beta-\sigma)(\lambda-\alpha)}{\mu+\lambda}\right]^{m}}{p(p-\delta)[\gamma p-\gamma+1]} \sqrt{a_{k} b_{k}} \leq 1 .
$$

Therefore (11) holds true if

$$
\sqrt{a_{k} b_{k}} \leq \frac{(k-\delta)(p-\chi)}{(p-\delta)(k-\chi)}
$$

for each $k \geq p+1, k, p \in \mathbb{N}$.

But (13) is satisfied if

$$
\frac{p(p-\delta)[\gamma p-\gamma+1]}{k(k-\delta)[\gamma k-\gamma+1]\left[\frac{\mu+\lambda+(k-p)(\beta-\sigma)(\lambda-\alpha)}{\mu+\lambda}\right]^{m}} \leq \frac{(k-\delta)(p-\chi)}{(p-\delta)(k-\chi)}
$$

or

$$
\begin{aligned}
\chi & \leq \frac{k p\left[(k-\delta)^{2}[\gamma k-\gamma+1]\left[\frac{\mu+\lambda+(k-p)(\beta-\sigma)(\lambda-\alpha)}{\mu+\lambda}\right]^{m}-(p-\delta)^{2}[\gamma p-\gamma+1]\right]}{k(k-\delta)^{2}[\gamma k-\gamma+1]\left[\frac{\mu+\lambda+(k-p)(\beta-\sigma)(\lambda-\alpha)}{\mu+\lambda}\right]^{m}-p(p-\delta)^{2}[\gamma p-\gamma+1]} \\
& =p\left[1-\frac{(k-p)(p-\delta)^{2}[\gamma p-\gamma+1]}{k(k-\delta)^{2}[\gamma k-\gamma+1]\left[\frac{\mu+\lambda+(k-p)(\beta-\sigma)(\lambda-\alpha)}{\mu+\lambda}\right]^{m}-p(p-\delta)^{2}[\gamma p-\gamma+1]}\right]
\end{aligned}
$$

Letting

$\Phi(k)=p\left[1-\frac{(k-p)(p-\delta)^{2}[\gamma p-\gamma+1]}{k(k-\delta)^{2}[\gamma k-\gamma+1]\left[\frac{\mu+\lambda+(k-p)(\beta-\sigma)(\lambda-\alpha)}{\mu+\lambda}\right]^{m}-p(p-\delta)^{2}[\gamma p-\gamma+1]}\right]$

we see that $\Phi(k)$ is increasing in $k$. This gives that

$$
\chi \leq p\left[1-\frac{(p-\delta)^{2}[\gamma p-\gamma+1]}{(p+1)(p+1-\delta)^{2}[\gamma k-\gamma+1]\left[\frac{\mu+\lambda+(\beta-\sigma)(\lambda-\alpha)}{\mu+\lambda}\right]^{m}-p(p-\delta)^{2}[\gamma p-\gamma+1]}\right] .
$$




\section{Growth And Distortion Theorems}

A growth and distortion property for function $f$ to be in the class $G_{n}^{p}(\alpha, \beta, \mu, \lambda, \gamma, \delta)$ is contained in the following theorem.

Theorem 3. If the function $f$ defined by (1) is in the class $G_{n}^{p}(\alpha, \beta, \mu, \lambda, \gamma, \delta)$, then for $|z|=r<1$, we have

$$
\begin{gathered}
r-\frac{|p(p-\delta)[\gamma p-\gamma+1]|}{(p+1)(p+1-\delta)[\gamma p+1]\left[\frac{\mu+\lambda+(\beta-\sigma)(\lambda-\alpha)}{\mu+\lambda}\right]^{m}} r^{2} \leq|f(z)| \leq \\
r+\frac{|p(p-\delta)[\gamma p-\gamma+1]|}{(p+1)(p+1-\delta)[\gamma p+1]\left[\frac{\mu+\lambda+(\beta-\sigma)(\lambda-\alpha)}{\mu+\lambda}\right]^{m}} r^{2}
\end{gathered}
$$

and

$$
\begin{gathered}
1-\frac{(p+1)|b|}{(p+1)(p+1-\delta)[\gamma p+1]\left[\frac{\mu+\lambda+(k-p)(\beta-\sigma)(\lambda-\alpha)}{\mu+\lambda}\right]^{m}} r \leq\left|f^{\prime}(z)\right| \leq \\
1+\frac{(p+1)|b|}{(p+1)(p+1-\delta)[\gamma p+1]\left[\frac{\mu+\lambda+(k-p)(\beta-\sigma)(\lambda-\alpha)}{\mu+\lambda}\right]^{m}} r .
\end{gathered}
$$

Proof. Since $f \in G_{n}^{p}(\alpha, \beta, \mu, \lambda, \gamma, \delta)$, from Theorem 1 readily yields the inequality

$$
\sum_{k=p+1}^{\infty} a_{k} \leq \frac{|p(p-\delta)[\gamma p-\gamma+1]|}{(p+1)(p+1-\delta)[\gamma p+1]\left[\frac{\mu+\lambda+(\beta-\sigma)(\lambda-\alpha)}{\mu+\lambda}\right]^{m}}
$$

Thus, for $|z|=r<1$, and making use of (14) we have

$|f(z)| \leq|z|+\sum_{k=p+1}^{\infty} a_{k}\left|z^{k}\right| \leq r+r^{2} \sum_{k=p+1}^{\infty} a_{k} \leq r+\frac{|p(p-\delta)[\gamma p-\gamma+1]|}{(p+1)(p+1-\delta)[\gamma p+1]\left[\frac{\mu+\lambda+(\beta-\sigma)(\lambda-\alpha)}{\mu+\lambda}\right]^{m}} r^{2}$

and

$|f(z)| \geq|z|-\sum_{k=p+1}^{\infty} a_{k}\left|z^{k}\right| \geq r-r^{2} \sum_{k=p+1}^{\infty} a_{k} \geq r-\frac{|p(p-\delta)[\gamma p-\gamma+1]|}{(p+1)(p+1-\delta)[\gamma p+1]\left[\frac{\mu+\lambda+(\beta-\sigma)(\lambda-\alpha)}{\mu+\lambda}\right]^{m}} r^{2}$.

Also from Theorem 1, it follows that

$$
\begin{gathered}
\frac{(p+1)(p+1-\delta)[\gamma p+1]\left[\frac{\mu+\lambda+(k-p)(\beta-\sigma)(\lambda-\alpha)}{\mu+\lambda}\right]^{m}}{p+1} \sum_{k=p+1}^{\infty} k a_{k} \leq \\
\left.\sum_{k=p+1}^{\infty} k(k-\delta)[\gamma k-\gamma+1]\right]\left[\frac{\mu+\lambda+(k-p)(\beta-\sigma)(\lambda-\alpha)}{\mu+\lambda}\right]^{m} a_{k} \leq|b| .
\end{gathered}
$$


M. Aljarrah, A. Aljarah, M. Darus - Fractional derivative operator ...

Hence

$\left|f^{\prime}(z)\right| \leq 1+\sum_{k=p+1}^{\infty} k a_{k}\left|z^{k}\right| \leq 1+r \sum_{k=p+1}^{\infty} k a_{k} \leq 1+\frac{(p+1)|b|}{(p+1)(p+1-\delta)[\gamma p+1]\left[\frac{\mu+\lambda+(k-p)(\beta-\sigma)(\lambda-\alpha)}{\mu+\lambda}\right]^{m}} r$

and

$\left|f^{\prime}(z)\right| \geq 1-\sum_{k=p+1}^{\infty} k a_{k}\left|z^{k}\right| \geq 1-r \sum_{k=p+1}^{\infty} k a_{k} \geq 1-\frac{(p+1)|b|}{(p+1)(p+1-\delta)[\gamma p+1]\left[\frac{\mu+\lambda+(k-p)(\beta-\sigma)(\lambda-\alpha)}{\mu+\lambda}\right]^{m}} r$.

This completes the proof of Theorem 3 .

\section{Closure Theorem}

Let the functions $f_{j}(z), j=1,2, \cdots, I$ be defined by

$$
f_{j}(z)=z-\sum_{k=p+1}^{\infty} a_{k, j} z^{k}, \quad a_{k, j} \geq 0
$$

for $z \in U$.

Closure theorems for the class $G_{n}^{p}(\alpha, \beta, \mu, \lambda, \gamma, \delta)$ are given by the following:

Theorem 4. Let the functions $f_{j}(z)$ defined by (15) be in the class $G_{n}^{p}(\alpha, \beta, \mu, \lambda, \gamma, \delta)$, $\alpha, \sigma \geq 0, \beta, \lambda, \mu>0, \lambda \neq \alpha$ and $m \in \mathbb{N}_{0}$, for every $j=1,2, \cdots, I$. Then the function $G(z)$ defined by

$$
G(z)=z-\sum_{k=p+1}^{\infty} p_{k} z^{k}, \quad p_{k} \geq 0
$$

is a member of the class $G_{n}^{p}(\alpha, \beta, \mu, \lambda, \gamma, \delta)$, where

$$
p_{k}=\frac{1}{I} \sum_{j=1}^{I} a_{k, j} \quad(k \geq 2) .
$$

Proof. Since $f_{j}(z) \in G_{n}^{p}(\alpha, \beta, \mu, \lambda, \gamma, \delta)$, it follows from Theorem 1 that

$\sum_{k=p+1}^{\infty} k(k-\delta)[\gamma k-\gamma+1]\left[\frac{\mu+\lambda+(k-p)(\beta-\sigma)(\lambda-\alpha)}{\mu+\lambda}\right]^{m} a_{k, j} \leq p(p-\delta)[\gamma p-\gamma+1]$

for every $j=1,2, \cdots, I$. 
M. Aljarrah, A. Aljarah, M. Darus - Fractional derivative operator ...

Hence,

$$
\begin{aligned}
\sum_{k=p+1}^{\infty} k & (k-\delta)[\gamma k-\gamma+1]\left[\frac{\mu+\lambda+(k-p)(\beta-\sigma)(\lambda-\alpha)}{\mu+\lambda}\right]^{m} p_{k} \\
& =\sum_{k=p+1}^{\infty} k(k-\delta)[\gamma k-\gamma+1]\left[\frac{\mu+\lambda+(k-p)(\beta-\sigma)(\lambda-\alpha)}{\mu+\lambda}\right]^{m}\left\{\frac{1}{I} \sum_{j=1}^{I} a_{k, j}\right\} \\
& =\frac{1}{I} \sum_{j=1}^{I}\left(\sum_{k=p+1}^{\infty} k(k-\delta)[\gamma k-\gamma+1]\left[\frac{\mu+\lambda+(k-p)(\beta-\sigma)(\lambda-\alpha)}{\mu+\lambda}\right]^{m} a_{k, j}\right) \\
& \leq \frac{1}{I} \sum_{j=1}^{I}|p(p-\delta)[\gamma p-\gamma+1]|=|p(p-\delta)[\gamma p-\gamma+1]|
\end{aligned}
$$

which implies that $G(z) \in G_{n}^{p}(\alpha, \beta, \mu, \lambda, \gamma, \delta)$.

Theorem 5. The class $G_{n}^{p}(\alpha, \beta, \mu, \lambda, \gamma, \delta)$ is closed under convex linear combination, where $\alpha, \sigma \geq 0, \beta, \lambda, \mu>0, \lambda \neq \alpha$ and $m \in \mathbb{N}_{0}$.

Proof. Suppose that the functions $f_{j}(z)(j=1,2)$ defined by $(15)$ are in the class $G_{n}^{p}(\alpha, \beta, \mu, \lambda, \gamma, \delta)$. It is suffices to prove that the function

$$
H(z)=\varphi f_{1}(z)+(1-\varphi) f_{2}(z) \quad(0 \leq \varphi \leq 1)
$$

is also in the class $G_{n}^{p}(\alpha, \beta, \mu, \lambda, \gamma, \delta)$.

Since, for $0 \leq \varphi \leq 1$,

$$
H(z)=z+\sum_{k=p+1}^{\infty}\left\{\varphi a_{k, 1}+(1-\varphi) a_{k, 2}\right\} z^{k},
$$

we observe that

$$
\begin{aligned}
\sum_{k=p+1}^{\infty} k & (k-\delta)[\gamma k-\gamma+1]\left[\frac{\mu+\lambda+(k-p)(\beta-\sigma)(\lambda-\alpha)}{\mu+\lambda}\right]^{m}\left\{\varphi a_{k, 1}+(1-\varphi) a_{k, 2}\right\} \\
& =\varphi \sum_{k=p+1}^{\infty} k(k-\delta)[\gamma k-\gamma+1]\left[\frac{\mu+\lambda+(k-p)(\beta-\sigma)(\lambda-\alpha)}{\mu+\lambda}\right]^{m} a_{k, 1} \\
& +(1-\varphi) \sum_{k=p+1}^{\infty} k(k-\delta)[\gamma k-\gamma+1]\left[\frac{\mu+\lambda+(k-p)(\beta-\sigma)(\lambda-\alpha)}{\mu+\lambda}\right]^{m} a_{k, 2} \\
& \leq \varphi|b|+(1-\varphi)|p(p-\delta)[\gamma p-\gamma+1]|=|p(p-\delta)[\gamma p-\gamma+1]| .
\end{aligned}
$$

Hence $H(z) \in G_{n}^{p}(\alpha, \beta, \mu, \lambda, \gamma, \delta)$. This completes the proof of Theorem 5 . 
M. Aljarrah, A. Aljarah, M. Darus - Fractional derivative operator ...

\section{Radil of Close-To-Convexity, Starlikeness and Convexity}

A function $f(z) \in \mathcal{A}$ is said to be close-to-convex of order $\eta$ if it satisfies

$$
\operatorname{Re}\left\{f^{\prime}(z)\right\}>\eta
$$

for some $\eta(0 \leq \eta \leq 1)$ and for all $z \in U$. Also a function $f(z) \in \mathcal{A}$ is said to be starlike of order $\eta$ if it satisfies

$$
\operatorname{Re}\left\{\frac{z f^{\prime}(z)}{f(z)}\right\}>\eta
$$

for some $\eta(0 \leq \eta \leq 1)$ and for all $z \in U$. Further, a function $f(z) \in \mathcal{A}$ is said to be convex of order $\eta$, if and only if $z f^{\prime}(z)$ is starlike of order $\eta$, that is if

$$
\operatorname{Re}\left\{1+\frac{z f^{\prime \prime}(z)}{f^{\prime}(z)}\right\}>\eta
$$

for some $\eta(0 \leq \eta \leq 1)$ and for all $z \in U$.

Theorem 6. If $f(z) \in G_{n}^{p}(\alpha, \beta, \mu, \lambda, \gamma, \delta)$, then $f(z)$ is close-to-convex of order $\eta$ in $|z|<h_{1}(p, \gamma, \delta, \eta)$, where

$h_{1}(p, \gamma, \delta, \eta)=\inf _{k}\left\{\frac{(p-\eta)(k-\delta)[\gamma k-\gamma+1]\left[\frac{\mu+\lambda+(k-p)(\beta-\sigma)(\lambda-\alpha)}{\mu+\lambda}\right]^{m}}{p(p-\delta)[\gamma p-\gamma+1]}\right\}^{\frac{1}{k}}(k, p \in \mathbb{N})$.

Proof. It is suffices to show that

$$
\left|\frac{f^{\prime}(z)}{z^{k}}-p\right| \leq \sum_{k=p+1}^{\infty} k a_{k}|z|^{k}<p-\eta
$$

and

$\sum_{k=p+1}^{\infty} k(k-\delta)[\gamma k-\gamma+1]\left[\frac{\mu+\lambda+(k-p)(\beta-\sigma)(\lambda-\alpha)}{\mu+\lambda}\right]^{m} a_{k} \leq p(p-\delta)[\gamma p-\gamma+1]$.

observe that (21) is true if

$$
\frac{k|z|^{k}}{p-\eta} \leq \frac{k(k-\delta)[\gamma k-\gamma+1]\left[\frac{\mu+\lambda+(k-p)(\beta-\sigma)(\lambda-\alpha)}{\mu+\lambda}\right]^{m}}{p(p-\delta)[\gamma p-\gamma+1]} .
$$


Solving (22) for $|z|$, we obtain

$$
|z| \leq\left\{\frac{(p-\eta)(k-\delta)[\gamma k-\gamma+1]\left[\frac{\mu+\lambda+(k-p)(\beta-\sigma)(\lambda-\alpha)}{\mu+\lambda}\right]^{m}}{p(p-\delta)[\gamma p-\gamma+1]}\right\}^{\frac{1}{k}},(k, p \in \mathbb{N})
$$

Theorem 7. If $f(z) \in G_{n}^{p}(\alpha, \beta, \mu, \lambda, \gamma, \delta)$, then $f(z)$ is starlike of order $\eta$ in $|z|<$ $h_{2}(p, \gamma, \delta, \eta)$, where

$h_{2}(p, \gamma, \delta, \eta)=\inf _{k}\left\{\frac{k(p-\eta)(k-\delta)[\gamma k-\gamma+1]\left[\frac{\mu+\lambda+(k-p)(\beta-\sigma)(\lambda-\alpha)}{\mu+\lambda}\right]^{m}}{p(k-\eta)(p-\delta)[\gamma p-\gamma+1]}\right\}^{\frac{1}{k}}(k, p \in \mathbb{N})$.

Proof. We must show that $\left|\frac{z f^{\prime}(z)}{f(z)}-p\right|<p-\eta$ for $|z|<h_{2}(p, \gamma, \delta, \eta)$. Since

$$
\left|\frac{z f^{\prime}(z)}{f(z)}-p\right| \leq \frac{\sum_{k=p+1}^{\infty}(k-p) a_{k}|z|^{k}}{1-\sum_{k=p+1}^{\infty} a_{k}|z|^{k}}
$$

if $\frac{(k-\eta)|z|^{k}}{p-\eta} \leq \frac{k(k-\delta)[\gamma k-\gamma+1]\left[\frac{\mu+\lambda+(k-p)(\beta-\sigma)(\lambda-\alpha)}{\mu+\lambda}\right]^{m}}{p(p-\delta)[\gamma p-\gamma+1]}, f(z)$ is starlike of order $\eta$.

Corollary 8. If $f(z) \in G_{n}^{p}(\alpha, \beta, \mu, \lambda, \gamma, \delta)$, then $f(z)$ is convex of order $\eta$ in $|z|<$ $h_{3}(p, \gamma, \delta, \eta)$

where

$h_{3}(p, \gamma, \delta, \eta)=\inf _{k}\left\{\frac{(p-\eta)(k-\delta)[\gamma k-\gamma+1]\left[\frac{\mu+\lambda+(k-p)(\beta-\sigma)(\lambda-\alpha)}{\mu+\lambda}\right]^{m}}{p(k-\eta)(p-\delta)[\gamma p-\gamma+1]}\right\}^{\frac{1}{k}}(k, p \in \mathbb{N})$.

\section{INTEGRAL OpERATORS}

In this section, we consider integral transforms of functions $f$ in the class $G_{n}^{p *}(\alpha, \beta, \mu, \lambda, \gamma, \delta)$. 
Theorem 9. If the function $f$ defined by (1) is in the class $G_{n}^{p *}(\alpha, \beta, \mu, \lambda, \gamma, \delta)$, where $\alpha, \sigma \geq 0, \beta, \lambda, \mu>0, \lambda \neq \alpha, m \in \mathbb{N}_{0}$. Then the function $F(z)$ defined by

$$
F(z)=\frac{c+1}{z^{c}} \int_{0}^{z} t^{c-1} f(t) d t, \quad(c>-1)
$$

also belongs to the class $G_{n}^{p *}(\alpha, \beta, \mu, \lambda, \gamma, \delta)$.

Proof. From (23), it follows that $F(z)=z-\sum_{k=p+1}^{\infty} r_{k} z^{n}$, where $r_{k}=\left(\frac{c+1}{c+k}\right) a_{k}$. Therefore

$$
\begin{aligned}
\sum_{k=p+1}^{\infty} k & (k-\delta)[\gamma k-\gamma+1]\left[\frac{\mu+\lambda+(k-p)(\beta-\sigma)(\lambda-\alpha)}{\mu+\lambda}\right]^{m} r_{k} \\
& =\sum_{k=p+1}^{\infty} k(k-\delta)[\gamma k-\gamma+1]\left[\frac{\mu+\lambda+(k-p)(\beta-\sigma)(\lambda-\alpha)}{\mu+\lambda}\right]^{m}\left(\frac{c+1}{c+k}\right) a_{k} \\
& \leq \sum_{k=p+1}^{\infty} k(k-\delta)[\gamma k-\gamma+1]\left[\frac{\mu+\lambda+(k-p)(\beta-\sigma)(\lambda-\alpha)}{\mu+\lambda}\right]^{m} a_{k}=|p(p-\delta)[\gamma p-\gamma+1]|,
\end{aligned}
$$

since $f(z) \in G_{n}^{p *}(\alpha, \beta, \mu, \lambda, \gamma, \delta)$. Hence by Theorem $1, F(z) \in G_{n}^{p *}(\alpha, \beta, \mu, \lambda, \gamma, \delta)$.

\section{Application in the Fractional Calculus}

Owa [11] gave the following definitions for the fractional calculus. For other difinitions, see $([12],[13],[14])$.

Definition 2. The fractional integral of order $\vartheta$ is defined by

$$
D_{z}^{-\vartheta} f(z)=\frac{1}{\Gamma(\vartheta)} \int_{0}^{z} \frac{f(t)}{(z-t)^{1-\vartheta}} d t
$$

where $\vartheta>0 . f(z)$ is analytic function in a simply connected region of the $z$-plane containing the origin and multiplicity of $(z-t)^{\vartheta-1}$ is remove by requiring $\log (z-t)$ to be real when $(z-t)>0$. 
Theorem 10. Let the function $f(z)$ be in the class $G_{n}(\mu, \delta, b)$. Then

$$
\begin{aligned}
& \left|D_{z}^{-\vartheta} f(z)\right| \leq \frac{p !}{\Gamma(p+\vartheta+1)}|z|^{p+\vartheta} \\
& {\left[1+\frac{p(p-\delta)[\gamma p-\gamma+1] \Gamma(p+2) \Gamma(p+\vartheta+1)}{\Gamma(p+1) \Gamma(p+\vartheta+2)(p+1)(p+1-\delta)[\gamma p+1]\left[\frac{\mu+\lambda+(\beta-\sigma)(\lambda-\alpha)}{\mu+\lambda}\right]^{m}}|z|^{k}\right]}
\end{aligned}
$$

and

$$
\begin{aligned}
& \left|D_{z}^{-\vartheta} f(z)\right| \geq \frac{p !}{\Gamma(p+\vartheta+1)}|z|^{p+\vartheta} \\
& {\left[1-\frac{p(p-\delta)[\gamma p-\gamma+1] \Gamma(p+2) \Gamma(p+\vartheta+1)}{\Gamma(p+1) \Gamma(p+\vartheta+2)(p+1)(p+1-\delta)[\gamma p+1]\left[\frac{\mu+\lambda+(\beta-\sigma)(\lambda-\alpha)}{\mu+\lambda}\right]^{m}}|z|^{k}\right] .}
\end{aligned}
$$

Proof. Using Theorem 1 we have

$$
\sum_{k=p+1}^{\infty} a_{k} \leq \frac{p(p-\delta)[\gamma p-\gamma+1]}{(p+1)(p+1-\delta)[\gamma p+1]\left[\frac{\mu+\lambda+(\beta-\sigma)(\lambda-\alpha)}{\mu+\lambda}\right]^{m}} .
$$

From Definition 2 we get

$$
\frac{D_{z}^{-\vartheta} f(z) \Gamma(p+\vartheta+1)}{p !}=z^{p}-\sum_{k=p+1}^{\infty} \frac{k ! \Gamma(p+\vartheta+1)}{p ! \Gamma(k+\vartheta+1)} a_{k} z^{k}
$$

and

$$
\begin{aligned}
\frac{D_{z}^{-\vartheta} f(z) \Gamma(p+\vartheta+1)}{p !} & =z^{p}-\sum_{k=p+1}^{\infty} \frac{\Gamma(k+1) \Gamma(p+\vartheta+1)}{\Gamma(p+1) \Gamma(k+\vartheta+1)} a_{k} z^{k} \\
& =z^{p}-\sum_{k=p+1}^{\infty} \Psi(k) a_{k} z^{k}
\end{aligned}
$$

where $\Psi(k)=\frac{\Gamma(k+1) \Gamma(p+\vartheta+1)}{\Gamma(p+1) \Gamma(k+\vartheta+1)}$.

We know that $\Psi(k)$ is a decreasing function of $k$ and $0<\Psi(k) \leq \Psi(p+1)=$ $\frac{\Gamma(p+2) \Gamma(p+\vartheta+1)}{\Gamma(p+1) \Gamma(p+\vartheta+2)}$. Using (28) and (29) we have

$$
\left|\frac{\Gamma(p+\vartheta+1) z^{-\vartheta} D_{z}^{-\vartheta} f(z)}{p !}\right| \leq|z|^{p}+\Psi(p+1)|z|^{p+1} \sum_{k=p+1}^{\infty} a_{k} \leq
$$


M. Aljarrah, A. Aljarah, M. Darus - Fractional derivative operator ...

$$
|z|^{p}+\frac{p(p-\delta)[\gamma p-\gamma+1] \Gamma(p+2) \Gamma(p+\vartheta+1)}{\Gamma(p+1) \Gamma(p+\vartheta+2)(p+1)(p+1-\delta)[\gamma p+1]\left[\frac{\mu+\lambda+(\beta-\sigma)(\lambda-\alpha)}{\mu+\lambda}\right]^{m}}|z|^{k},
$$

which gives (2). We also have

$$
\begin{gathered}
\left|\frac{\Gamma(p+\vartheta+1) z^{-\vartheta} D_{z}^{-\vartheta} f(z)}{p !}\right| \geq|z|^{p}-\Psi(p+1)|z|^{p+k} \sum_{k=p+1}^{\infty} a_{k} \geq \\
|z|^{p}-\frac{p(p-\delta)[\gamma p-\gamma+1] \Gamma(p+2) \Gamma(p+\vartheta+1)}{\Gamma(p+1) \Gamma(p+\vartheta+2)(p+1)(p+1-\delta)[\gamma p+1]\left[\frac{\mu+\lambda+(\beta-\sigma)(\lambda-\alpha)}{\mu+\lambda}\right]^{m}}|z|^{k}
\end{gathered}
$$

which gives (10).

Acknowledgements. The work here is supported by UKM grant: GUP-2017064 .

\section{REFERENCES}

[1] A.A. Amourah and F. Yousef, Some Properties of a Class of Analytic Functions Involving a New Generalized Differential Operator, Boletim da Sociedade Paranaense de Matematica, 22 (2017), 1-9.

[2] A. Aljarah and M. Darus, Differential sandwich theorems for p-valent functions involving a generalized differential operator, Far East Journal of Mathematical Sciences, 96 (2015), 651-660.

[3] M. Darus and R. W. Ibrahim, On subclasses for generalized operators of complex order, Far East J. Math. Sci., 33(3) (2009), 299-308.

[4] S. F. Ramadan and M. Darus, On the Fekete-Szego inequality for a class of analytic functions defined by using generalized differential operator, Acta Universitatis Apulensis, 26 (2011), 167-78.

[5] M. Darus and I. Faisal, Problems and properties of a new differential operator, Journal of Quality Measurement and Analysis (JQMA), 7( 1) (2011), 41-51.

[6] G. S. Salagean, Subclasses of univalent functions, In Complex Analysis-Fifth Romanian-Finnish Seminar, pp. 362-372. Springer Berlin Heidelberg, 1983.

[7] S. R. Swamy, Inclusion properties of certain subclasses of analytic functions, Int. Math. Forum, 7(36) (2012), 1751-1760.

[8] F. M. Al-Oboudi, On univalent functions defined by a generalized Sălăgean operator, International Journal of Mathematics and Mathematical Sciences, 27 (2004), 1429-1436. 
M. Aljarrah, A. Aljarah, M. Darus - Fractional derivative operator ...

[9] L. Xiao-Fei and W. An-Ping, A subclass of analytic functions with negative coefficients, International Journal of Pure and Applied Mathematics, 78(1)(2012), 75-83.

[10] M. Kamali and S. Akbulut, On a subclass of certain convex functions with negative coefficients, Applied Mathematics and Computation, 145(2)(2003), 341-350.

[11] S. Owa, On the distortion theorems I, Kyungpook Math. J., 18(1)(1978), 53-59.

[12] H. M. Srivastava and S. Owa, A new class of analytic functions with negative coefficients, Commentarii Mathematici Universitatis Sancti Pauli, 35(2)(1986), 175188.

[13] H. M. Srivastava, M. Saigo and S. Owa, A class of distortion theorems involving certain operators of fractional calculus, Journal of Mathematical Analysis and Applications, 131(2)(1988), 412-420.

[14] K. Nishimoto, Fractional derivative and integral, Part I, J. College Engrg. Nihon Univ., B-17(1976), 11-19.

Maymoona Aljarrah

School of Mathematical Sciences, Faculty of Science and Technology, Universiti Kebangsaan Malaysia, Bangi, Selangor, Malaysia

email: Maimoonah1981@gmail.com

Anas Aljarah

School of Mathematical Sciences, Faculty of Science and Technology, Universiti Kebangsaan Malaysia, Bangi, Selangor, Malaysia

email:anasjrah@yahoo.com

Maslina Darus

School of Mathematical Sciences, Faculty of Science and Technology, Universiti Kebangsaan Malaysia, Bangi, Selangor, Malaysia email: maslina@ukm.edu.my (Corresponding author) 